\title{
Mitochondria: the panacea to improve oocyte quality?
}

\author{
Lingbin $\mathrm{Qi}^{1 \#}$, Xian Chen ${ }^{2 \#}$, Jian Wang ${ }^{1}$, Bo Lv ${ }^{1}$, Junhui Zhang ${ }^{3}$, Bin $\mathrm{Ni}^{4}$, Zhigang Xue ${ }^{1,5}$ \\ ${ }^{1}$ Department of Regenerative Medicine, Tongji University School of Medicine, Shanghai 200092, China; ${ }^{2}$ Shenzhen Key Laboratory for \\ Reproductive Immunology of Peri-implantation, Shenzhen Zhongshan Institute for Reproduction and Genetics, Fertility Center, Shenzhen \\ Zhongshan Urology Hospital, Shenzhen 518045, China; ${ }^{3}$ Reproductive Medical Center, Department of Obstetrics and Gynecology, the First \\ Affiliated Hospital of Anhui Medical University, Hefei 230032, China; ${ }^{4}$ NHC Key Laboratory of Birth Defect for Research and Prevention, Hunan \\ Provincial Maternal and Child Health Care Hospital, Changsha 410008, China; ${ }^{5}$ Reproductive Medicine Center, Tongji Hospital, Tongji University \\ School of Medicine, Shanghai 200065, China \\ Contributions: (I) Conception and design: L Qi, X Chen, Z Xue; (II) Administrative support: B Ni, Z Xue; (III) Provision of study materials or \\ patients: L Qi, J Wang; (IV) Collection and assembly of data: B Lv, J Zhang; (V) Data analysis and interpretation: L Qi, X Chen; (VI) Manuscript \\ writing: All authors; (VII) Final approval of manuscript: All authors. \\ \#These authors contributed equally to this work. \\ Correspondence to: Zhigang Xue. Department of Regeneration Medicine, Translation Center of Stem Cell Research, Tongji Hospital, Tongji \\ University School of Medicine, Shanghai, 200065, China. Email: xuezg@tongji.edu.cn; Bin Ni. Hunan Provincial Maternal and Child Health Care \\ Hospital, NHC Key Laboratory of Birth Defect for Research and Prevention, Changsha 410008, China. Email: nibin2007@163.com.
}

\begin{abstract}
Oocyte quality is one of the most important factors involving in female reproduction. The number of compromised oocytes will increase with maternal age, while mitochondrial dysfunction has implicated in age-related poor oocyte. Together with the successful application of ooplasmic transfer (OT) and the critical role of mitochondria in the oocyte, functional mitochondria transfer may be a feasible strategy to improve oocyte quality. However, limitation on ethics and laws are strictly and optimal condition or methods to exert transferring need to be further explored. Therefore, the role of oocyte mitochondria and the effective molecular involving in oocyte quality will be hot topics in next few years. In this review, we summarize the potential mechanism of mitochondria in oocyte and embryo development and discuss the next step for mitochondrial transfer therapy.
\end{abstract}

Keywords: Mitochondria; mitochondrial replacement therapy; mitochondrial transfer therapy; infertility; oocyte quality

Submitted Jul 12, 2019. Accepted for publication Nov 14, 2019.

doi: $10.21037 /$ atm.2019.12.02

View this article at: http://dx.doi.org/10.21037/atm.2019.12.02

\section{Introduction}

Infertility has become a growing problem worldwide, and decreased oocyte quality mainly culminates in the agerelated deterioration of reproductive capacity (1). Network of regulating oocyte quality is greatly complicated and mechanism is still unclear, but it's certain clear that energy metabolism and competing endogenous RNA (ceRNA) regulating network are hugely essential (2).

Attributed to the key role in regulation of cellular metabolism and epigenetics, mitochondria have been paid more attentions in recent years (3-6). Several hypotheses implicate that mitochondria are one of the critical indicators in oocyte quality (7). Mitochondrial functions are important for the early embryo development and implantation, including the formation of meiotic spindles and the maintenance of the metaphase II (MII) spindle before fertilization (8). All of the complex processes oocyte goes through prior to ovulation and fertilization require energy, especially those adenosine triphosphate (ATP) derived from mitochondrial oxidative phosphorylation (OXPHOS) (9). It has been shown that insufficient ATP production during the oogenesis and embryogenesis will result in aneuploidy, a condition in which chromosomal segregation errors are frequently encountered (10). Furthermore, mitochondria as the center of cellular metabolism will have an impact on 
gene expression by cross-talk with nucleus (11). Interaction between mitochondria and nucleus can involve in many biological progresses which are important for both oocyte maturation and embryonic development (11-13). Autologous mitochondrial transfer technologies (AUGMENT, one of strategy in assisted reproduction technology) which transfer mitochondria from oocyte precursor cells to MII oocyte have succeeded in promoting outcome (14). Subsequently, clinical research also indicates that mitochondrial transfer actually can rejuvenate ageing oocyte (15).

However, a recent randomized pilot trial finds AUGMENT had no significant curative effect on ameliorating oocyte quality (16). This result strong strikes the previous study and give us more consideration about the credibility of mitochondrial transfer therapy. Therefore, what bioprocesses happen after transferring and why reverse results can be received by the similar operations will be highly concerned in the next few years. Here, we will describe the potential mechanism for mitochondria involving in oocyte quality and the future of mitochondriarelated therapy.

\section{Mitochondrial dynamics: power to maintain normal oocyte and embryonic development}

In human, quantity of mitochondria suffer from a great variation during oocyte maturation. Mitochondria in mature oocyte have been increased 1,000 times from only few dozens to more than 100 thousand ones comparing to primary follicles $(17,18)$. Then, mitochondria are averagely distributed to each blastomere during embryonic development, but the total number is relatively stable before the blastocyst stage (19). Likewise, mitochondrial activity is also relatively stable before the blastocyst stage. Although reasons of this appearance are still obscure, disorder of mitochondria activation can certainly cause serious oocyte dysplasia $(20,21)$. Mitochondria in mammalian oocytes are transcriptionally and bioenergetically silent (22), especially in immature eggs (23). This quiescent state is believed to be important to keep minimal mitochondrial DNA (mtDNA) mutations, because these mutations will then be passed down to the embryo (24). The energy required for oocyte maturation is mainly provided by surrounding granulosa cells and cumulus cells $(25,26)$. Along with the accomplishment of fertilization and the ensuring embryonic development, mitochondria-derived energy gradually come to dominate $(9,27,28)$. When the embryo develops to the blastocyst stage, mitochondria in the embryo have become slender, the structure of ridges is intact, and the mtDNA is largely replicated, indicating that mitochondria have completed the transition from quiescence to activation $(29,30)$. Thus, mitochondrial dynamics are involved in protecting oocyte from oxidative damage and support early embryonic development. Decline in oocyte mitochondrial reserve will trigger the energic shortage during preimplantation which may induce embryo arrested. For the purpose of rescuing poor oocyte, injection of function mitochondria during intracytoplasmic sperm injection may be effective to compensate maternal mitochondria deficient, then reconstructing mitochondrial activation.

\section{Mitochondrial metabolites: affect oocyte quality on epigenetic modification}

Increasing evidences have demonstrated that age-related decline in oocyte quality partly ascribes to alter epigenetic modification (11,31-33) (Figure 1). The inducements of epigenetic alteration are intricate, but the role of mitochondria in these progresses is worth being focused on. Actually, previous data has revealed that metabolites produced by mitochondria could remodel chromatin then regulating genetic expression (11). For instance, citrate could be cracked into acetyl-CoA which would donate the acetyl groups for histone acetyltransferases (HATs) (34). Certainly, content of acetyl-CoA is mitochondriadependent in most parts. When mitochondrial metabolism is high, acetyl-CoA levels will increase, then advancing histone acetylation to drive gene expression by chromatin loose or vice versa (35). Mitochondrial generated $\alpha$-Ketoglutarate $(\alpha-K G)$ is also a crucial accessory factor of histone methylation. It could be a key co-factor to initiate the function of histone demethylases (HDMs) to identify special substrates and undock methyl groups from histones (36). Simultaneously, S-adenosyl methionine (SAM) engages most parts of histone methyltransferases (HMTs) to activate methyl group transfer (37). And synthesis of SAM relies on folate cycle and ATP which are both controlled by mitochondria. Importantly, ATP-dependent chromatin remodeling complexes require mitochondrial ATP to support necessary energy for chromatin modulating, then binding to genes specially (38). Previous research found levels of SAM is a key regulator for naïve-primed transition of human embryonic stem cells (hESCs) (39). This data imply that mitochondria are critical in modifying epigenetics on human early embryos and even oocytes. 


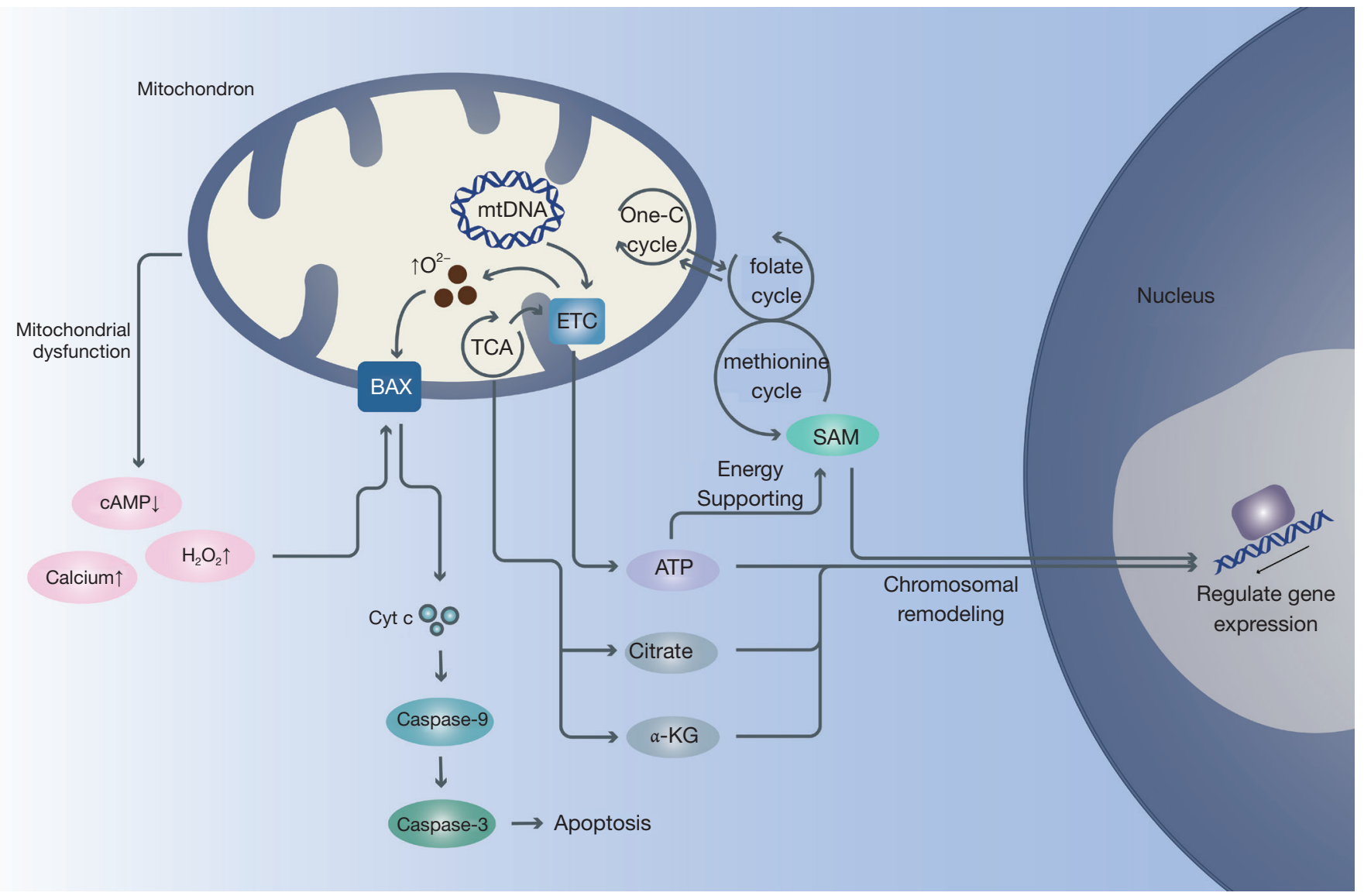

Figure 1 The role of mitochondria in metabolism, apoptosis and gene expression. For caspase activation by mitochondria, multiple signal factors (such as $\mathrm{Ca}^{2+}$, cAMP and ROS) can modify permeability of mitochondrial membrane by activating Bax then releasing cyt $\mathrm{c}$ from mitochondria into the cytosol. Thereby, cyt c triggers caspase-9 and initiates the proteolytic cascade that culminates in apoptosis. Simultaneously, caspase-8 can also activate Bax by slicing Bid, which results in the similar apoptosis pathway. Additionally, mitochondria could provide enough energy for cell by synthesize ATP via ETC. Mitochondrial metabolites of TCA cycle (such as ATP, -KG and citrate) could also regulate gene expression via remodeling chromatin. ATP synthesis is dependent on mitochondria oxidative phosphorylation driven by the ETC and ATP synthase. SAM is one of metabolites of methionine and folate cycles that supported by mitochondrial onecarbon cycle. While SAM synthesis requires ATP to support necessary energy for chromatin modulating and then binding to genes specially. mtDNA, mitochondrial DNA; ETC, electron transport chain; TCA, tricarboxylic acid cycle; SAM, S-adenosyl methionine; cAMP, cyclic adenosine monophosphate; ATP, adenosine triphosphate; cyt c, cytochrome c; $\alpha$-KG, $\alpha$-ketoglutaric acid.

Experience on mouse reveals that lysine acetyltransferase 8 (KAT8, one kind of HATs and depend on acetyl-CoA) is essential for oocyte maturation and follicular reserved (40). What's more, mitochondrial heteroplasmy might increase the risk of preterm birth by interaction with nucleus (41). These evidences clue us that mitochondrial dysfunction and mtNDA mutation are likely to affect the expression of nuclear genes by modifying the whole epigenetic landscape then inducing many extremely serious consequences in oocyte and pre-plantation development.

\section{Mitochondria-related signaling pathway: threshold of oocyte survivals}

The amounts of matured oocyte in each menstrual cycle is controlled by apoptosis strictly, where only less than one percent germ cells can strive into survival at last (42). Mitochondria play a critical role in apoptosis via regulating many signal factors involving in apoptosis such as $\mathrm{Ca}^{2+}$, cyclic adenosine monophosphate (cAMP), reactive oxygen species (ROS), etc. (43) (Figure 1). There are two major 




Figure 2 Technological process of mitochondrial transfer therapy (AUGMENT). Mitochondria were isolated from autologous cells germlines such as granular and cumulus cells, oogonial stem cells or mesenchymal stem cells from diverse tissues, and injected into oocyte with a sperm by ICSI to form a reconstructed zygote. ICSI, intracytoplasmic sperm injection.

mechanisms in apoptosis, the intrinsic and the extrinsic pathways (44). For the intrinsic pathway, mitochondrial membrane permeabilization (MMP) can be altered by apoptosis signals then releasing pro-apoptotic factors such as cytochrome c (cyt c) from mitochondria $(45,46)$. As a result, apoptosis-dependent effectors such as caspase- 3 will be activated and fragment nucleus DNA then resulting in apoptosis. Calcium is one of the most important factors in cellular homeostasis, which can be regulated by mitochondria. Mitochondria can absorb calcium by anion-selective channels or transporters to maintain calcium homeostasis (47). When mitochondria dysfunction happen, balance of calcium will be broken out and high concentration of $\mathrm{Ca}^{2+}$ in cytoplasm will disturb MMP then inducing apoptosis (48). And relationship between oocyte ageing and calcium homeostasis mess has been identified in mice (49). Additionally, mitochondrial dysfunction will increase the production of ROS. Increasing ROS level have been identified strong relationship with oocyte ageing, which can intensify apoptosis in oocyte (50) and lower fertilization rates (51). What's more, mitochondria remain relatively low activity until developing to the blastocyst stage proposal for reducing superfluous ROS (52). It has been identified that healthy cells can rescue apoptotic cells via transferring mitochondria (53). Coincidentally, mitochondrial transfer has capacity to provide against oxidative stress-mediated mitochondrial dysfunction (54). Looking ahead, it's possible to presume that replenishing mitochondria in a suitable degree might be a selective method to cope with mitochondrial decline in ageing oocyte.

\section{Practice of the "mitochondrial therapy": overhastiness}

Based on the well therapeutic use by ooplasmic transfer (OT) (55), we know that compromised oocyte may have deficiency in ooplasmics (56). Additionally, preciousness of benign MII oocyte and problem of "3 parents babies" both press people to find out the effective ingredient in ooplasmics. According to the context, mitochondria are the most expected targets. If assumed, transferring autogenous mitochondria (AUGMENGT) is suitable to improve oocyte quality for avoiding above problem caused by OT (57). The general processes of AUGMENGT are that: (I) prepare autologous cell germlines such as oogonial stem cells, granulosa cells or mesenchymal stem cells from diverse tissue; (II) isolate mitochondria from them; (III) inject isolated mitochondria to MII oocyte by microinjection (Figure 2). This technology is led by OvaScience Inc., who annotated they had rescued poor oocyte by AUGMENT in 2014 (58). Since emergence of AUGMENT, trends of following were raised overwhelming the world rapidly. Observations from some international fertility clinics show that the successful rates of AUGMENT range from $25 \%$ to $53 \%$ and pregnancy rates are increased greatly (between 3 - and 18-fold increase compared to control group) (59). There is no doubt that it's a bold attempt and may be good news for those people with poor oocyte quality. But in some ways, this strategy seems too much radical. It's unadvisable to experience this technology on patients in the absence of firm support from completed basic and clinical trials. A recent prospective 
study demonstrated that AUGMENT technique does not seem to improve embryo quality in infertile patients with premature ovarian ageing and a background of poor embryo quality in previous in vitro fertilization (IVF) cycles. Moreover, there were no significantly difference in the ratio of euploid embryos obtained per injected MII and per fertilized oocyte between experimental and control groups (16). So far, Food and Drug Administration of American has forbidden this technology to perform on clinic, and the pioneer, OvaScience, have turn to join other researches (59).

Generally speaking, AUGMENT has some advantages. Firstly, compared with nucleus transfer, AUGMENT can overcome the inherited bottleneck which is in case of making so-called "three parents" baby. Secondly, transferring functional mitochondria to sluggish oocyte could provide enough energy for them to develop as normal. And diverse mitochondrial-related metabolites can be adjusted to the regular levels. What's more, cell survival pathway is proposed to be activated after transferring, then initiating the quiescence mitochondria and prevent oocyte from apoptosis. However, this technology now is still immature. On one hand, autologous stem cells are uneasy to acquire. More importantly, technologies for isolating and authenticating functional mitochondria from cell cultures are puerile. It's difficult for us to analyze the number and completed function of isolated mitochondria, while the dosage and quality of transferring mitochondria are definitive to the success. Previous studies demonstrated that overfull replication of mtDNA could reduce early embryo activity and implantation potential (60). On another hand, the detailed internal mechanism of this therapy is now yet not clear. It's unclear about how the complementary mitochondria work in low-quality oocyte and how mitochondria cooperate with nucleus to regulate cellular proliferation and to refresh oocyte. Thus, it is recommended that mitochondrial transfer therapy is unsuitable to use for curing infertility in current conditions, and the efficacy and safety of this technology are need to be investigated further.

\section{Perspectives and conclusion}

Beyond mitochondrial replacement therapy (i.e., nucleus transfer), much interest in this field has concerned on AUGMNET as a potential therapeutic option for rescuing compromised oocyte. The development of this technology seems rough, but it's only limited on clinical application.
Basic research of discovering the relationship between mitochondria and oocyte quality and potential effective factors involving in $\mathrm{OT}$ are much more intriguing. What's more, sufficient evidences on basic researches is fundamental to advance AUGMENT in stability and efficiency. Emerging data suggest that mitochondrial metabolism is significantly important to prevent oocyte from death (61), but directly injecting purified mitochondria doesn't work well. The reasons for failure may be attributed to the following points: (I) exfoliate mitochondria from endoplasmic reticulum (ER) which is essential to keep mitochondrial function (62); (II) too much cell debris containing in isolated mitochondria may cause overload metabolism in poor oocyte; (III) discard multiple other functional small molecular that is potential to benefit oocyte quality. Therefore, advancing in the technologies of isolating and optimizing scheme of transferring may make revolution in this scope. Once its effectiveness and safety are appropriately confirmed, mitochondrial transfer therapies will have great potential to be used as a novel treatment in assisted reproduction. Although alternative and innovative approaches are being proposed, it still remains in a very early stage. Nevertheless, search for an effective solution to improve oocyte quality will be continuous.

\section{Acknowledgments}

We gratefully thank the anonymous referees for their important and helpful comments.

Funding: This work was supported by National Natural Science Foundation of China (No. 81771651), Science and Technology Commission of Shanghai Municipality (No. 16JC1404700) and Hunan Provincial Science and Technology Research Fund (2019JJ80078).

\section{Footnote}

Conflicts of Interest: The authors have no conflicts of interest to declare.

Ethical Statement: The authors are accountable for all aspects of the work in ensuring that questions related to the accuracy or integrity of any part of the work are appropriately investigated and resolved.

\section{References}

1. Cecchino GN, Seli E, Alves da Motta EL, et al. The role 
of mitochondrial activity in female fertility and assisted reproductive technologies: overview and current insights. Reprod Biomed Online 2018;36:686-97.

2. Susor A, Kubelka M. Translational Regulation in the Mammalian Oocyte. Results Probl Cell Differ 2017;63:257-95.

3. D'Aquila P, Bellizzi D, Passarino G. Mitochondria in health, aging and diseases: the epigenetic perspective. Biogerontology 2015;16:569-85.

4. Schroeder EA, Raimundo N, Shadel GS. Epigenetic silencing mediates mitochondria stress-induced longevity. Cell Metab 2013;17:954-64.

5. Carden T, Singh B, Mooga V, et al. Epigenetic modification of miR-663 controls mitochondria-to-nucleus retrograde signaling and tumor progression. J Biol Chem 2017;292:20694-706.

6. Spinelli JB, Haigis MC. The multifaceted contributions of mitochondria to cellular metabolism. Nat Cell Biol 2018;20:745-54.

7. Schatten H, Sun QY, Prather R. The impact of mitochondrial function/dysfunction on IVF and new treatment possibilities for infertility. Reprod Biol Endocrinol 2014;12:111.

8. Benkhalifa M, Ferreira YJ, Chahine H, et al. Mitochondria: participation to infertility as source of energy and cause of senescence. Int J Biochem Cell Biol 2014;55:60-4.

9. Ben-Meir A, Burstein E, Borrego-Alvarez A, et al. Coenzyme Q10 restores oocyte mitochondrial function and fertility during reproductive ageing. Aging Cell 2015;14:887-95.

10. May-Panloup P, Boucret L, Chao de la Barca JM, et al. Ovarian ageing: the role of mitochondria in oocytes and follicles. Hum Reprod Update 2016;22:725-43.

11. Matilainen O, Quiros PM, Auwerx J. Mitochondria and Epigenetics - Crosstalk in Homeostasis and Stress. Trends Cell Biol 2017;27:453-63.

12. Ge ZJ, Schatten H, Zhang CL, et al. Oocyte ageing and epigenetics. Reproduction 2015;149:R103-14.

13. Whidden L, Martel J, Rahimi S, et al. Compromised oocyte quality and assisted reproduction contribute to sex-specific effects on offspring outcomes and epigenetic patterning. Hum Mol Genet 2016;25:4649-60.

14. Couzin-Frankel J. Eggs unlimited. Science 2015;350:620-4.

15. Huang R, Fang C, Jia L, et al. Mitochondrial Transfer from Autologous Bone Marrow Mesenchymal Stem Cells Improves Oocyte Quality. Fertil Steril 2018;110:e190.

16. Labarta E, de Los Santos MJ, Herraiz S, et al. Autologous mitochondrial transfer as a complementary technique to intracytoplasmic sperm injection to improve embryo quality in patients undergoing in vitro fertilization-a randomized pilot study. Fertil Steril 2019;111:86-96.

17. Jansen RP, de Boer K. The bottleneck: mitochondrial imperatives in oogenesis and ovarian follicular fate. Mol Cell Endocrinol 1998;145:81-8.

18. Tang JJ, Ying P, Shao JY, et al. Oocyte Mitochondiral Aging__Bottleneck of Assisted Reproductive Technology(ART). Reproduction \& Contraception 2013;33:255-60.

19. St John JC, Facucho-Oliveira J, Jiang Y, et al. Mitochondrial DNA transmission, replication and inheritance: a journey from the gamete through the embryo and into offspring and embryonic stem cells. Hum Reprod Update 2010;16:488-509.

20. Zhao L, Lu T, Gao L, et al. Enriched endoplasmic reticulum-mitochondria interactions result in mitochondrial dysfunction and apoptosis in oocytes from obese mice. J Anim Sci Biotechnol 2017;8:62 .

21. Zhang Y, ShiYang X, Zhang Y, et al. Exposure to aristolochic acid I compromises the maturational competency of porcine oocytes via oxidative stress-induced DNA damage. Aging (Albany NY) 2019;11:2241-52.

22. Allen JF, de Paula WB. Mitochondrial genome function and maternal inheritance. Biochem Soc Trans 2013;41:1298-304.

23. Bentov Y, Yavorska T, Esfandiari N, et al. The contribution of mitochondrial function to reproductive aging. J Assist Reprod Genet 2011;28:773-83.

24. de Paula WB, Agip AN, Missirlis F, et al. Female and male gamete mitochondria are distinct and complementary in transcription, structure, and genome function. Genome Biol Evol 2013;5:1969-77.

25. Motta PM, Nottola SA, Makabe S, et al. Mitochondrial morphology in human fetal and adult female germ cells. Hum Reprod 2000;15 Suppl 2:129-47.

26. Collado-Fernandez E, Picton HM, Dumollard R. Metabolism throughout follicle and oocyte development in mammals. Int J Dev Biol 2012;56:799-808.

27. He JY, Mao CC, Reyes A, et al. The AAA(+) protein ATAD3 has displacement loop binding properties and is involved in mitochondrial nucleoid organization. J Cell Biol 2007;176:141-6.

28. Leese HJ, Barton AM. Pyruvate and glucose uptake by mouse ova and preimplantation embryos. J Reprod Fertil 1984;72:9-13.

29. St John J. The control of mtDNA replication during 
differentiation and development. Biochim Biophys Acta 2014;1840:1345-54.

30. Hashimoto S, Morimoto N, Yamanaka M, et al. Quantitative and qualitative changes of mitochondria in human preimplantation embryos. J Assist Reprod Genet 2017;34:573-80.

31. Chamani IJ, Keefe DL. Epigenetics and Female Reproductive Aging. Front Endocrinol (Lausanne) 2019;10:473.

32. Nie J, Xiao P, Wang X, et al. Melatonin prevents deterioration in quality by preserving epigenetic modifications of porcine oocytes after prolonged culture. Aging (Albany NY) 2018;10:3897-909.

33. Titus S, Stobezki R, Oktay K. Impaired DNA Repair as a Mechanism for Oocyte Aging: Is It Epigenetically Determined? Semin Reprod Med 2015;33:384-8.

34. Montgomery DC, Sorum AW, Guasch L, et al. Metabolic Regulation of Histone Acetyltransferases by Endogenous Acyl-CoA Cofactors. Chem Biol 2015;22:1030-9.

35. Menzies KJ, Zhang H, Katsyuba E, et al. Protein acetylation in metabolism - metabolites and cofactors. Nat Rev Endocrinol 2016;12:43-60.

36. Kooistra SM, Helin K. Molecular mechanisms and potential functions of histone demethylases. Nat Rev Mol Cell Biol 2012;13:297-311.

37. Teperino R, Schoonjans K, Auwerx J. Histone Methyl Transferases and Demethylases; Can They Link Metabolism and Transcription? Cell Metab 2010;12:321-7.

38. Hota SK, Bruneau BG. ATP-dependent chromatin remodeling during mammalian development. Development 2016;143:2882-97.

39. Sperber H, Mathieu J, Wang Y, et al. The metabolome regulates the epigenetic landscape during naive-to-primed human embryonic stem cell transition. Nat Cell Biol 2015;17:1523-35.

40. Yin S, Jiang X, Jiang H, et al. Histone acetyltransferase KAT8 is essential for mouse oocyte development by regulating reactive oxygen species levels. Development 2017;144:2165-74.

41. Crawford N, Prendergast D, Oehlert JW, et al. Divergent Patterns of Mitochondrial and Nuclear Ancestry Are Associated with the Risk for Preterm Birth. J Pediatr 2018;194:40-6. e4.

42. Tilly JL. Commuting the death sentence: How oocytes strive to survive. Nat Rev Mol Cell Biol 2001;2:838-48.

43. Tiwari M, Prasad S, Tripathi A, et al. Apoptosis in mammalian oocytes: a review. Apoptosis 2015;20:1019-25.

44. Verbrugge I, Johnstone RW, Smyth MJ. SnapShot:
Extrinsic apoptosis pathways. Cell 2010;7:1192, 1192.e1-2.

45. Tripathi A, Chaube SK. High cytosolic free calcium level signals apoptosis through mitochondria-caspase mediated pathway in rat eggs cultured in vitro. Apoptosis 2012;17:439-48.

46. Su Z, Yang Z, Xu Y, et al. Apoptosis, autophagy, necroptosis, and cancer metastasis. Mol Cancer 2015;14:48.

47. Xu Z, Zhang D, He X, et al. Transport of Calcium Ions into Mitochondria. Curr Genomics 2016;17:215-9.

48. Giorgi C, Baldassari F, Bononi A, et al. Mitochondrial $\mathrm{Ca}(2+)$ and apoptosis. Cell Calcium 2012;52:36-43.

49. Takahashi T, Igarashi H, Kawagoe J, et al. Poor Embryo Development in Mouse Oocytes Aged In Vitro Is Associated with Impaired Calcium Homeostasis. Biol Reprod 2009;80:493-502.

50. Chaube SK, Prasad PV, Thakur SC, et al. Hydrogen peroxide modulates meiotic cell cycle and induces morphological features characteristic of apoptosis in rat oocytes cultured in vitro. Apoptosis 2005;10:863-74.

51. Takahashi T, Takahashi E, Igarashi H, et al. Impact of oxidative stress in aged mouse oocytes on calcium oscillations at fertilization. Mol Reprod Dev 2003;66:143-52.

52. Leese HJ. Metabolism of the preimplantation embryo: 40 years on. Reproduction 2012;143:417-27.

53. Wang X, Gerdes HH. Transfer of mitochondria via tunneling nanotubes rescues apoptotic PC12 cells. Cell Death Differ 2015;22:1181-91.

54. Jiang D, Gao F, Zhang Y, et al. Mitochondrial transfer of mesenchymal stem cells effectively protects corneal epithelial cells from mitochondrial damage. Cell Death Dis 2016;7:e2467.

55. Cohen J, Scott R, Alikani M, et al. Ooplasmic transfer in mature human oocytes. Mol Hum Reprod 1998;4:269-80.

56. Barritt J, Willadsen S, Brenner C, et al. Cytoplasmic transfer in assisted reproduction. Hum Reprod Update 2001;7:428-35.

57. Woods DC, Tilly JL. Autologous Germline Mitochondrial Energy Transfer (AUGMENT) in Human Assisted Reproduction. Semin Reprod Med 2015;33:410-21.

58. Hardymon GF, Nicholas T, Stuart T, et al. OvaScience. Harvard Business School Case 815-058, September 2014.

59. Lee K. An Ethical and Legal Analysis of Ovascience - A Publicly Traded Fertility Company and its Lead Product AUGMENT. Am J Law Med 2018;44:508-28.

60. Diez-Juan A, Rubio C, Marin C, et al. Mitochondrial DNA content as a viability score in human euploid 
embryos: less is better. Fertil Steril 2015;104:534-41.e1.

61. Sansone P, Savini C, Kurelac I, et al. Packaging and transfer of mitochondrial DNA via exosomes regulate escape from dormancy in hormonal therapy-resistant breast cancer. Proc Natl Acad Sci U S A

Cite this article as: Qi L, Chen X, Wang J, Lv B, Zhang J, Ni B, Xue Z. Mitochondria: the panacea to improve oocyte quality? Ann Transl Med 2019;7(23):789. doi: 10.21037/atm.2019.12.02
2017;114:E9066-75.

62. Lewis SC, Uchiyama LF, Nunnari J. ER-mitochondria contacts couple mtDNA synthesis with mitochondrial division in human cells. Science 2016;353:aaf5549. 